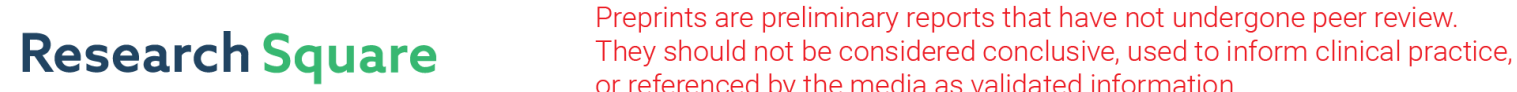 or referenced by the media as validated information. \\ Rural-urban disparities in unmet long-term care needs and the demand for community care services among elderly people in China
}

Yanbing Zeng

Xiamen University

Lixia Wang

Xiamen University

Liangwen Zhang

Xiamen University

Ya Fang ( $\nabla$ fangya@xmu.edu.cn)

Xiamen University School of Public Health

Research article

Keywords: unmet needs, long-term care, community care services, elderly people, China

Posted Date: October 7th, 2019

DOI: https://doi.org/10.21203/rs.2.15656/v1

License: (c) (i) This work is licensed under a Creative Commons Attribution 4.0 International License.

Read Full License 


\section{Rural-urban disparities in unmet long-term care needs and the demand for community care services among elderly people in China}

Yanbing Zeng ${ }^{1,2}$, Lixia Wang 1,2, Liangwen Zhang ${ }^{1,2}$, and Ya Fang 1,2, *

Author Note

1. State Key Laboratory of Molecular Vaccinology and Molecular Diagnostics, School of Public Health, Xiamen University, Xiamen, Fujian, China

2. Key Laboratory of Health Technology Assessment of Fujian Province University,

School of Public Health, Xiamen University, Xiamen, Fujian, China

* Corresponding author: Ya Fang

Address: School of Public Health, Xiamen University, Xiang'an Nan Road,

Xiang'an District, Xiamen, Fujian, China, 361102.

Telephone: (+86)0592-2880636

Fax: (+86)0592-2880639

Email: fangya@xmu.edu.cn

Email addresses of other authors:

Yanbing Zeng: ybingzeng@163.com

LixiaWang: alisiawang@163.com

Liangwen Zhang: 1095553765@qq.com 


\section{Abstract}

Background: Estimates of unmet needs, as an indicator of future needs for long-term care (LTC) services, have become increasingly crucial policy concerns. This study aimed to examine the urban-rural differences in unmet needs and the demand for community care service among community-dwelling elderly people in China.

Methods: The data come from the 2014 Chinese Longitudinal Health Longevity Survey (CLHLS). A total of 1587 community residents aged $65+$ with disabilities in the activities of daily living (ADLs) were included in this study. Based on the Andersen theoretical model, binary logistic regression was used to estimate the correlates of unmet needs in LTC. A chi-square test was used to examine the differences in expected needs for community-based LTC services between urban and rural areas.

Results: Over half (55.07\%) of the participants reported their needs were unmet. Poor economic status and reluctant caregivers seriously affected elderly unmet needs. Among urban older adults, those who were male and lonely reported more unmet needs. Among rural ones, those with severe ADL disability and poor self-rated health reported more unmet needs. In addition, access to medication and home visit services were negatively associated with unmet needs. Living with children (69.12\%) was viewed as the most desirable living arrangement among older adults, while living in a LTC facility seemed to be more accepted for rural residents with unmet needs than for other elderly respondents. Residents showed a high demand for community LTC care 
services, with $82.55 \%$ of them expecting to need home visits and $74.29 \%$ to healthcare education. Specifically, rural residents had greater expected needs for every community care service than their urban counterparts. However, only $4.66 \%$ to $36.42 \%$ of the respondents reported that all eight types of services were available, which was far below the demand for these services.

Conclusion: The risk of having unmet LTC needs is largely determined by elderly people's economic status and caregivers' willingness to provide care for both rural and urban elderly residents. More attention should be paid to psychological consulting services in urban areas, as well as personal care, home visits, psychological consulting and healthcare education services in rural areas.

Keywords: unmet needs, long-term care, community care services, elderly people, China

\section{Background}

According to the National Bureau of Statistics of China, there were approximately 158.5 million Chinese people aged 65 or older at the end of 2017 , accounting for $11.4 \%$ of the total population. The disability rate in older adults aged over 60 years was $7.0 \%$, and the rate increased with age according to the China Comprehensive Geriatric Assessment Study in 2012[1]. Enormous long-term care (LTC) needs are in the future for current and future elderly populations in China [2,3]. However, the burden of traditional family care for elderly individuals in China is mounting. In cities, the 
emerging "4-2-1" family structure (four grandparents; two parents, neither of whom has siblings; and one child) is an ominous development. In the countryside, due to the massive out-migration of young people to cities for work, the fulfillment of filial piety by adult children is increasingly challenging[4,5]. Although China has experienced rapid economic development, it is still limited in the improvement of institutional buildings and social service infrastructure to support adequate LTC for older people[6].

Long-term care needs primarily refer to a range of formal and informal aids and services that individuals may require for assistance or care with the basic activities of daily living (ADLs), such as eating, dressing and bathing[3, 7]. It has been shown that nearly half of those with unmet needs may not be able to eat or may be burned or scarred while bathing, which could significantly diminish these individuals' quality of life[8]. Using national panel data, a study indicated that older Chinese adults with unmet needs had an approximately $10 \%$ increased risk of mortality compared with those whose needs were met when demographic data were controlled[7]. The presence of unmet needs also leads to negative events, such as emergency room visits, nursing home placement, and hospitalization $[9,10]$. Estimates of unmet needs may serve as an indicator of future needs for LTC services $[10,11]$.

The probabilities and correlates of unmet LTC needs among older adults have been a growing concern of many scholars. The overall proportion of unmet needs for the basic activities of daily living among elderly individuals aged 65 years or older 
was evaluated to be from $22 \%$ in the United States to $61.4 \%$ in China[7,12]. The rate among people aged 60 years and above in Brazil was 18\%, which was slightly lower than the rate in the previous countries [13]. Among elderly individuals who were eligible for community-based care through Medicare or Medicaid, 58\% had some unmet needs [14]. Some studies have indicated that the prevalence rates of adverse consequences of unmet needs are twice as high among older adults with elevated depressive symptoms than among those without depression [15].

Many studies have indicated that low educational attainment, low income, not being currently married, living alone, severe ADL disability, cognitive impairment, and unavailability and unaffordability of services were significantly associated with unmet LTC needs $[8,13,16-18]$. Other studies have found differences between urban and rural residents. Among the oldest elderly people, economic status, someone other than a family member being the primary caregiver, caregivers' willingness to provide care, timely medication, self-rated health, and self-rated life satisfaction were significantly associated with unmet needs for both urban and rural residents; however, age, the son and daughter-in-law being the primary caregiver, severe ADL disability, expected access to community-based care services, and optimism were only significant for urban residents, and gender and cognitive function were only significant for rural residents[3]. For those aged 45 years and older, people with rural hukou had a higher chance of experiencing unmet LTC needs irrespective of whether they lived in rural or urban regions[2]. 
Although previous studies have recognized the factors and the adverse consequences of unmet LTC needs among older adults, limitations still exist. On the one hand, few studies have focused on the urban-rural differences of unmet needs among community-dwelling people aged 65 years and over, let alone in China. On the other hand, attention to the implications of LTC needs for community care services among the elderly population is limited. Taking traditional filial piety into consideration, policy makers are increasingly turning to community-based care to bridge the growing gap between LTC care needs and provision in China, which has limited caregiving resources. To reduce the burden of unmet needs and to facilitate the development and targeting of community-based LTC care services, it is important to determine the correlates of unmet needs and the supply and demand of community care services in urban and rural areas in China. Therefore, this study aimed to explore rural-urban disparities in unmet LTC needs and expected community care service needs among community-dwelling elderly people aged 65 years and older in China.

\section{Methods}

\section{Study sample}

The study sample was from the 2014 wave, the most recent wave, of the Chinese Longitudinal Healthy Longevity Survey (CLHLS), approved by the Institutional Review Board of Duke University Medical Center. The CLHLS, initiated in 1998, is the first high-quality, national longitudinal survey of Chinese residents aged 80+ and 
has been conducted every two or three years since its inception. Older people aged 65-79 have been included 2002. In the 2014 wave, 7192 respondents were interviewed by trained interviewers accompanied by a doctor, nurse, or medical school student who performed a basic health examination for every participant in his/her home. The CLHLS provides a rich set of information on demographic and socioeconomic characteristics, physical and psychological function, health-related behaviors and lifestyles, and health care. In addition, information on the care needs of the population with functional limitations and self-reported unmet LTC needs is also collected. A detailed description of the design, sample distribution and content of the data collected from CLHLS can be found in Zeng et a l(2016)[19].

We focused on community residents who were aged $65+$ at the time of the interview and had disabilities in performing at least one of the six ADLs (bathing, dressing, toileting, in-door transferring, maintaining continence, and eating). For the purpose of this study, we excluded participants who were under 65 years old $(n=85)$ or institutionalized $(n=198)$, those who had incomplete residential information $(n=173)$ and those who did not have any ADL disability $(\mathrm{n}=5149)$. The data from the resulting sample of 1587 (23.56\%) participants were analyzed (Figure 1).

\section{Measures}

\section{Unmet long-term care needs}

Measures of long-term care needs vary across studies, and currently, there is still no established standard for assessing these needs[7]. In this study, long-term care needs 
were measured by the assistance needed in performing any of the six ADL tasks (eating, dressing, bathing, getting in/out of bed, indoor transferring, and toileting). If a respondent required assistance in any of the six tasks in his or her daily life, he or she was considered to have long-term care needs; if not, he or she was considered to not have long-term care needs. Unmet needs were measured by the question, "Does the assistance provided by caregivers meet your needs?" The answers were dichotomized into fully met (coded 0) and not fully met (coded 1) needs. Although this measure is based on an individual's perception of his or her need for any assistance, there is evidence that it is a valid indicator of unmet needs[20].

\section{Independent variables}

We focused our analysis on different factors of unmet long-term care needs between urban residents and rural residents, including predisposing factors, enabling resources, and need factors based on the Andersen theoretical model [21]. Predisposing factors included age, gender, ethnicity (Han versus Non-Han), years of education ( 0 versus 1-6 versus $>6$ ), occupation before 60 (farmer versus nonfarmer), exercise (yes versus no) and involvement in social activity (yes versus no).

Enabling resources included three subsets of variables: economics, caregiving resources and community-based care service availability. Measures of economics included financial independence (yes versus no), economic status compared to their neighbors (good versus fair versus bad). Measures of caregiving resources included availability of adequate medication when needed (yes versus no), currently married 
(yes versus no), living alone (yes versus no), primary caregiver for ADL assistance (spouse versus daughter and son-in-law versus son and daughter-in-law versus others) and their willingness to provide care (yes versus no), and the total cost of care paid in the past week ( 0 versus $1-300$ versus $301+$ ). The availability of care service indicated whether personal care and home visits were offered in the community.

Need variables referred to severe ADL disability (unable to perform at least three of the six ADL tasks) (yes versus no), cognitive impairment (Mini-Mental Status Examination score lower than 24) [22](yes versus no), self-rated health (good versus fair versus bad), self-rated life satisfaction (good versus fair versus bad), participant loneliness (yes versus no) and participant depression (yes versus no).

\section{Statistical analysis}

First, we examined the overall prevalence of unmet LTC needs and the differences between groups by a chi-square test or $t$-test as appropriate. Second, for the analysis of the role of urban vs. rural residence, we used two binary logistic models to examine the relationship between unmet LTC needs and the predisposing, enabling and needs factors. Furthermore, the chi-square test was used to determine the differences in expectations regarding future living arrangements and community-based care needs by residence. In addition, differences in community-based service supply were also examined. Variables with missing data were multiple imputed by FCS. All analyses were performed using SAS version 9.4 software (SAS Institute, Inc., Cary, NC, USA). 


\section{Results}

\section{Sample characteristics}

Table 1 presents the characteristics of the study sample. Overall, among the 1587 community-dwelling participants, over half $(55.07 \%)$ reported that their needs for LTC were unmet. Their average age was 92.78 years old. In addition, $64.34 \%$ of the sample was female, $4.98 \%$ was of non-Han nationality, and $69.69 \%$ had never attended school. The participants attended social activities regularly but did not exercise as often. Compared to the urban residents, the rural residents were older, less educated, exercised less and were more likely to be unsatisfied with their needs being met.

In terms of enabling resources, although a great majority $(98.93 \%)$ of the participants were financially dependent, more than two-thirds $(68.87 \%)$ reported that their economic status was similar to that of their neighbors. The participants lived mainly with others, particularly in urban areas. Children, especially sons and daughters-in-law, were identified as the primary caregivers both in urban and rural areas, indicating that family care is still the primary form of care in China. Fortunately, over $93 \%(93.01 \%)$ of the caregivers were willing to take care of them. However, some noticeable rural-urban differences existed. The elderly participants in urban areas had better access to medication and personal care but not to home visits. In addition, the participants living in rural areas were more likely to not be married and to live alone. 
In addition, the elderly participants were generally in poor health, both physically and mentally. Nearly half (49.02\%) of them were unable to perform at least three of the six ADL tasks, and 42.03\% rated their life satisfaction as bad. The rural elderly participants were more likely to have cognitive impairment as well as feelings of loneliness, while their urban counterparts were prone to report depression.

\section{Unmet LTC needs and factors}

Table 2 shows the odds ratios between the unmet needs of the elderly residents and various variables, including the predisposing, enabling and needing factors, based on binary logistic regression both in urban and rural areas. In these two areas, residents' economic status and willingness of their caregivers seriously affected unmet needs. The poorer the economic status of the elderly participant was, the greater the possibility that his or her needs were unmet, and the same relationship was found for caregivers' unwillingness to provide care.

In urban areas, men had greater odds of having unmet needs than women $(\mathrm{OR}=1.55,95 \% \mathrm{CI}=1.06-2.28)$, and lonely participants also reported more unmet needs than participants who did not report loneliness ( $\mathrm{OR}=1.58,95 \% \mathrm{CI}=1.13-2.21)$. In rural areas, people with severe ADL disability $(\mathrm{OR}=1.73,95 \% \mathrm{CI}=1.21-2.47)$ and poorer self-rated health $(\mathrm{OR}=1.40,95 \% \mathrm{CI}=1.16-1.70)$ were more likely to report their needs as unsatisfied. Moreover, greater access to medication $(\mathrm{OR}=0.25,95 \%$ $\mathrm{CI}=0.09-0.67)$ and home visits $(\mathrm{OR}=0.50,95 \% \mathrm{CI}=0.36-0.70)$ was negatively related 
to unmet needs in rural areas.

\section{Living arrangement and community care service need expectations}

Table 3 shows the expectations regarding living arrangements and community care service needs among the community-dwelling elderly participants with LTC needs in urban and rural areas. Overall, living with children (69.12\%) was the most desirable living arrangement for older people. Nearly one quarter (23.13\%) wanted to live alone (or with a spouse) with children living nearby. The minority (6.93\%) preferred to live alone no matter where their children lived, and less than $1 \%(0.82 \%)$ chose to lead a life in an LTC facility. However, for people with unmet needs, LTC facilities seemed to be more accepted, especially in rural areas.

The residents showed a high demand for community care services (54.88\%-82.55\%). The greatest demand was for home visits $(82.55 \%)$, followed by healthcare education (74.29\%) and psychological consulting (64.90\%). The three services with the lowest demand were daily shopping (54.88\%), legal aid (58.03\%) and neighborhood relations $(58.48 \%)$. In addition, rural residents had greater expected community care service needs than their urban counterparts. Rural residents with unmet needs had a stronger demand for personal care and psychological consulting than other participants. However, among urban residents, whether their needs were met, their expected needs for community care services were equally high.

\section{Availability of community services}


Table 4 shows the availability of care service in the communities where the elderly participants lived. The rates of services available were generally low, ranging from $4.66 \%$ to $36.42 \%$. The greatest supply was for healthcare education $(38.06 \%)$, followed by home visits $(36.42 \%)$, which was similar to the demand. Overall, the availability of home visit and daily shopping services in rural areas was significantly higher than that in urban areas, while the opposite was true for the availability of psychological consulting services. In the rural communities, the rates of availability of home visit, psychological consulting and healthcare education services in the communities where people whose needs were met lived were markedly higher than in the communities where the people with unmet needs lived. However, for urban areas, there were no significant differences in these two groups.

\section{Discussion}

The findings from this study provide national information about unmet LTC needs among community-dwelling older adults and their expectations regarding eight types of community care services. The study demonstrates that the prevalence of unmet LTC needs among community-dwelling older adults in need of assistance in ADLs is high in China. Rural-urban differences exist in the influencing factors of unmet LTC needs and elderly individuals' expectations regarding their needs for community care services. 


\section{Prevalence of unmet LTC needs}

This study shows that nearly $23.56 \%$ of community-living older adults aged 65 and over are in need of long-term care for their basic activities of daily living, and 55.07\% of them report their needs as unmet in China, which is a rate that is clearly higher than that of other countries. For instance, the proportion of unmet needs has been reported to be $22 \%$ among older adults aged 65 years or older living in communities in noninstitutional housing in the USA [12]. However, some studies have indicated this proportion may have been underestimated because respondents who received assistance were not asked whether that help was adequate or sufficient in the National Long-Term Care Survey in the USA $[9,23]$. Another study reported that the prevalence of BADL disability among elderly people aged 65 and over in Brazil was $16.0 \%$ and that $47.0 \%$ of those people with disabilities had their needs met, while only $10.3 \%$ did not [13]. In that study, unmet needs were defined as the presence of at least one unavailable need for BADLs. That is, in this $47.0 \%$ of the respondents, many might have been able to obtain help but were not fully satisfied. Moreover, some indicated that the prevalence of unmet needs among elderly people who required assistance in any of the six tasks for more than 3 months in China was even higher than that of this study, reaching 61.4\%[7]. Furthermore, older adults in rural areas had a higher rate of unmet needs than their urban counterparts, which is in line with other studies [16,24]. Despite the different measures of unmet LTC needs, we can see that China, especially rural areas of China, faces great challenges in terms of 
unmet LTC needs among older people.

\section{Rural-urban differences in the influencing factors of unmet LTC needs}

The results of this study indicated that the willingness of caregivers and economic status were significantly important for both urban and rural residents, similar to the prior studies $[3,8,25]$. Regardless of the caregiver's identity, the willingness of caregivers to provide care is the most influential factor with the largest OR changes. The quality of care provided by unwilling caregivers is questionable. According to Maslow's hierarchy of needs theory, respect plays an important role in human needs[26]. If elderly individuals need to be taken care of, but the caregiver is not willing to provide the service, the elderly individuals are likely to feel that they are not respected and not valued, especially in China where filial piety is valued. Consequently, care services are difficult to satisfy. That is, service attitudes remain important in elder care, although most of caregivers are elderly people's children. In addition, elderly people with a good economic status generally have greater access to medical resources and are better able to pay for health care services, thus lowering their risk of having unmet needs[3, 13].

Feelings of loneliness were positively associated with the risk of having unmet needs, but living alone was not among urban older residents, which is different from prior studies $[13,27]$. Loneliness is believed to be a common and serious problem for the elderly population[28]; loneliness is defined as a deficiency between an 
individual's actual and desired social relationships, resulting in feelings of distress, dissatisfaction or detachment [29-31]. Previous studies have shown that older people who live alone have a higher chance of having unmet needs because they lack the strongest support from family [13,27]. That is, a lack of family support, rather than living alone, leads to unmet needs. After retirement, the social networks of urban residents narrow substantially, and their main caregivers, their children, are usually too busy to take care of them. Urban older adults are likely to feel lonely and consequently unsatisfied with their care services. Therefore, more attention should be paid to the loneliness of urban older adults, reducing it through the provision of more support.

In contrast to urban residents, rural-dwelling older adults who were unable to access timely medication or home visits, had severe ADL disability and had poor self-rated health were more likely to have unmet LTC needs, which is consistent with previous studies[24]. Higher levels of dependency are associated with unmet needs [16,32]; however, for rural disabled residents, the availability of both qualified formal and informal care services is inadequate to meet their needs. First, in China, compared to urban areas, rural areas have lower population densities, poorer infrastructure, fewer qualified hospitals and nursing homes. Although rural residents reported the availability of more home visit services, the service quality of rural community health centers is also not as good as that of urban community health centers. Therefore, it is difficult for rural residents to obtain good formal care services. Second, informal care 
has been decreasing due to rural-urban migration [33]. Many working age adults flock to cities from the countryside for a better job or a more modern living environment, leaving elderly people behind; thus, more rural elderly people live alone than urban elderly people, as shown in Table 1.

\section{Community care service needs and planning for the future}

In accordance with the previous results, China is facing a growing gap between diversified LTC needs and provision, both in urban and rural areas [5, 34]. Although the resources and infrastructure for elderly care are limited, most Chinese elderly people prefer to age in place, either co-residing with children or living nearby. The findings from the study indicated that the quantity of the eight kinds of community care services, such as personal care and home visits, that were in demand from the participants was approximately 2 to 13 times the quantity supplied in 2014 . Whether or not the LTC needs were satisfied, the supply of personal care and psychological consulting services were far below the demand. Home visit services were most needed, followed by healthcare education services, although both of these types of services were the most accessible. More emphasis should be placed on community care services, in partnership with family and institutional services.

In addition, rural older adults need more community care services than their urban counterparts, and their specific needs vary, similar to the findings of other studies [34]. As reported above, for urban residents, loneliness is significantly 
associated with unmet needs, while for rural residents, available medical services and health are a greater problem. Therefore, to decrease the possibility of unmet needs, more psychological consulting services are needed in urban areas, while personal care, home visits, psychological consulting and healthcare education services are needed in rural areas.

\section{Limitations}

This study has several limitations. First, the sample population is too old. The CLHLS initially investigated elderly people aged 80 and above, and although elderly people aged 65-79 have been included since 2002, the sample mainly represents the oldest elderly people in China, which may cause selection bias. Second, the cross-sectional design of the study could limit its ability to capture causal relationships among variables. Third, the measurement of unmet needs based on the self-reported lack of assistance for ADLs may lead to underestimation of unmet needs, although this measurement is widely used. Further studies should find a better way to improve the precision and multiplicity of analysis.

\section{Conclusions}

Urban-rural disparities in unmet LTC needs among older adults have been a particular concern for researchers and policy makers because of the negative effects of unmet LTC needs on health and quality of life. The risk of having unmet needs associated 
with ADL disability in LTC is largely determined by elderly people's economic status and caregivers' willingness to provide care for both rural and urban elderly people. There is a need for an overall improvement in the planning, provision and financing of long-term care services for elderly individuals in China. Community care services, as the best way to meet the needs of the elderly population, face serious challenges in China. To uphold traditional filial piety, policy makers, based on urban-rural planning, should increasingly turn to community care to bridge the growing gap between care needs and provision. For example, day care centers, nursing homes, activity centers, mutual support service centers and day care services should be provided in all urban and rural communities. Furthermore, more attention should be paid to psychological consulting services in urban areas, as well as personal care, home visits, psychological consulting and healthcare education services in rural areas. Meanwhile, family members are still likely the best available care providers because of the inadequate professional, skilled nursing care services in recent years, so more training courses should be offered to family members to improve their caregiving skills.

\author{
Abbreviations \\ LTC, long-term care; CLHLS, Chinese Longitudinal Health Longevity Survey ; ADL, \\ activities of daily living; FCS, fully conditional specification; SAS, Statistics Analysis \\ System; NC,; USA, United States of America; ORs, Odds Ratios; CIs, Confidence \\ Intervals.
}




\section{Declarations}

\section{Ethics approval and consent to participate}

The data used in our research is secondary data. The survey of the data named "CLHLS" already obtained the ethical approval and informed consent, and was approved by research ethics committees of Duke University and Peking University (IRB00001052-13074). The data used in this study was anonymised before its use.

\section{Consent for publication}

Not applicable.

\section{Availability of data and material}

The secondary data CLHLS have been used in this study. This data set is freely available in this website:

http://opendata.pku.edu.cn/dataverse/CHADS?q=\&types=datasets\&sort=dateSort\&or der $=$ desc $\&$ page $=1$

\section{Competing interests}

The authors declare that they have no competing interests.

\section{Funding}

This study was funded by the National Natural Science Foundation of China [Grant Numbers. 81573257, 71874147], the Natural Science Foundation of Fujian Province of China [Grant Numbers. 2017J01133]. 


\section{Authors' contributions}

YF conceived and designed the study, supervised the data analysis; YZ, LW and LZ wrote the paper; YZ performed all statistical analyses and LW contributed to revising the paper. The authors listed meet the authorship criteria according to the latest guidelines of the International Committee of Medical Journal Editors, and all are in agreement with the manuscript.

\section{Acknowledgments}

We would like to thank the Center for Healthy Aging and Development Studies, Peking University for supporting this database. Data used for this research was provided by the study entitled 'Chinese Longitudinal Healthy Longevity Survey' (CLHLS) managed by the Center for Healthy Aging and Development Studies, Peking University. CLHLS is supported by funds from the U.S. National Institutes on Aging (NIA), China Natural Science Foundation, China Social Science Foundation, and UNFPA. 


\section{References}

1. Ma LN, Li ZZ, Tang Z, Sun F, Diao LJ, Li J, He Y, Dong BR, Li Y: Prevalence and socio-demographic characteristics of disability in older adults in China: Findings from China Comprehensive Geriatric Assessment Study. Archives of Gerontology and Geriatrics 2017, 73:199-203.

2. Zhu YM, Osterle A: Rural-urban disparities in unmet long-term care needs in. China: The role of the hukou status. Social Science \& Medicine 2017, 191:30-37.

3. Zhu HY: Unmet needs in long-term care and their associated factors among the oldest old in China. BMC Geriatrics 2015, 15.

4. Flaherty JH, Liu ML, Ding L, Ding BR, Ding QF, Li X, Xiao SF: China: The aging giant. $J$ Am Geriatr Soc 2007, 55(8):1295-1300.

5. Feng ZL, Liu C, Guan XP, Mor V: China's Rapidly Aging Population Creates Policy Challenges In Shaping A Viable Long-Term Care System. Health Aff 2012, 31(12):2764-2773.

6. Lei $\mathrm{P}$, Feng $\mathrm{Z}, \mathrm{Wu} \mathrm{Z}$ : The availability and affordability of long-term care for disabled older people in China: The issues related to inequalities in social security benefits. Archives of Gerontology \& Geriatrics 2016, 67:21.

7. Zhen Z, Feng Q, Gu D: The Impacts of Unmet Needs for Long-Term Care on Mortality Among Older Adults in China. Journal of Disability Policy Studies 2015, 25(4):243-251.

8. Desai MM, Lentzner HR, Weeks JD: Unmet need for personal assistance with activities of daily living among older adults. Gerontologist 2001, 41(1):82-88.

9. Gaugler JE, Kane RL, Kane RA, Newcomer R: Unmet care needs and key outcomes in dementia. J Am Geriatr Soc 2005, 53(12):2098-2105.

10. Long SK, King J, Coughlin TA: The implications of unmet need for future health care use: Findings for a sample of disabled medicaid beneficiaries in New York. Inquiry-J Health Care Organ Provis Financ 2005, 42(4):413-420.

11. Tennstedt $\mathrm{S}$, McKinlay J, Kasten L: UNMET NEED AMONG DISABLED ELDERS - A PROBLEM IN ACCESS TO COMMUNITY LONG-TERM-CARE. Social Science \& Medicine 1994, 38(7):915-924.

12. Xu HP, Covinsky KE, Stallard E, Thomas J, Sands LP: Insufficient Help for Activity of Daily Living Disabilities and Risk of All-Cause Hospitalization. J Am Geriatr Soc 2012, 60(5):927-933.

13. de Andrade TB, de Andrade FB: Unmet need for assistance with activities of daily life among older adults in Brazil. Rev Saude Publica 2018, 52:9.

14. Komisar HL, Feder J, Kasper JD: Unmet long-term care needs: An analysis of medicare-medicaid dual eligibles. Inquiry-J Health Care Organ Provis 
Financ 2005, 42(2):171-182.

15. Xiang XL, An RP, Heinemann A: Depression and Unmet Needs for Assistance With Daily Activities Among Community-Dwelling Older Adults. Gerontologist 2018, 58(3):428-437.

16. Liu YH, Chang HJ, Huang CC: The Unmet Activities of Daily Living (ADL) Needs of Dependent Elders and their Related Factors: An Approach from Both an Individual- and Area-Level Perspective. Int J Gerontol 2012, 6(3):163-168.

17. Momtaz YA, Hamid TA, Ibrahim R: Unmet needs among disabled elderly Malaysians. Social Science \& Medicine 2012, 75(5):859-863.

18. Berridge C, Mor V: Disparities in the Prevalence of Unmet Needs and Their Consequences Among Black and White Older Adults. Journal of Aging and Health 2018, 30(9):1427-1449.

19. Yi Z, Feng Q, Gu D, Vaupel J: Demographics, Phenotypic Health Characteristics and Genetic Analysis of Centenarians in China. Mechanisms of Ageing \& Development 2016, 165.

20. Morrow-Howell N, Proctor E, Rozario P: How much is enough? Perspectives of care recipients and professionals on the sufficiency of in-home care. Gerontologist 2001, 41(6):723-732.

21. Andersen RM: REVISITING THE BEHAVIORAL-MODEL AND ACCESS TO MEDICAL-CARE - DOES IT MATTER. J Health Soc Behav 1995, 36(1):1-10.

22. Zhong BL, Chen SL, Tu X, Conwell Y: Loneliness and Cognitive Function in Older Adults: Findings From the Chinese Longitudinal Healthy Longevity Survey. Journals of Gerontology Series B-Psychological Sciences and Social Sciences 2017, 72(1):120-128.

23. Allen SM, Mor V: The prevalence and consequences of unmet need Contrasts between cider and younger adults with disability. Med Care 1997, 35(11):1132-1148.

24. $\mathrm{Hu} \mathrm{B}$, Wang J: Unmet long-term care needs and depression: The double disadvantage of community-dwelling older people in rural China. Health \& Social Care in the Community 2019, 27(1):126-138.

25. Peng $\mathrm{R}, \mathrm{Wu} \mathrm{B}$, Ling $\mathrm{L}$ : Undermet Needs for Assistance in Personal Activities of Daily Living Among Community-Dwelling Oldest Old in China From 2005 to 2008. Res Aging 2015, 37(2):148-170.

26. Maslow AH: Motivation and personality. Quarterly Review of Biology 1970(1):187-202.

27. Burchardt T, Jones E, Obolenskaya P: Formal and Informal Long-Term Care in the Community: Interlocking or Incoherent Systems? J Soc Policy 2018, 47(3):479-503.

28. Heylen L: The older, the lonelier? Risk factors for social loneliness in old age. Ageing \& Society 2010, 30(7):1177-1196. 
29. Zhou Z, Wang P, Fang Y: Loneliness and the risk of dementia among older Chinese adults: gender differences. Aging \& Mental Health 2018, 22(4):519-525.

30. Victor CR, Scambler SJ, Bowling A, Bond J: The prevalence of, and risk factors for, loneliness in later life: a survey of older people in Great Britain. Ageing \& Society 2005, 25(3):357-375.

31. Sorkin D, Rook KS, Lu JL: Loneliness, lack of emotional support, lack of companionship, and the likelihood of having a heart condition in an elderly sample. Annals of Behavioral Medicine 2002, 24(4):290-298.

32. Newcomer R, Kang T, LaPlante M, Kaye S: Living quarters and unmet need for personal care assistance among adults with disabilities. Journals of Gerontology Series B-Psychological Sciences and Social Sciences 2005, 60(4):S205-S213.

33. Guo M, Aranda MP, Silverstein M: The impact of out-migration on the inter-generational support and psychological wellbeing of older adults in rural China. Ageing \& Society 2009, 29:1085-1104.

34. Zhou J, Walker A: The need for community care among older people in China. Ageing \& Society 2016, 36(6):1312-1332.

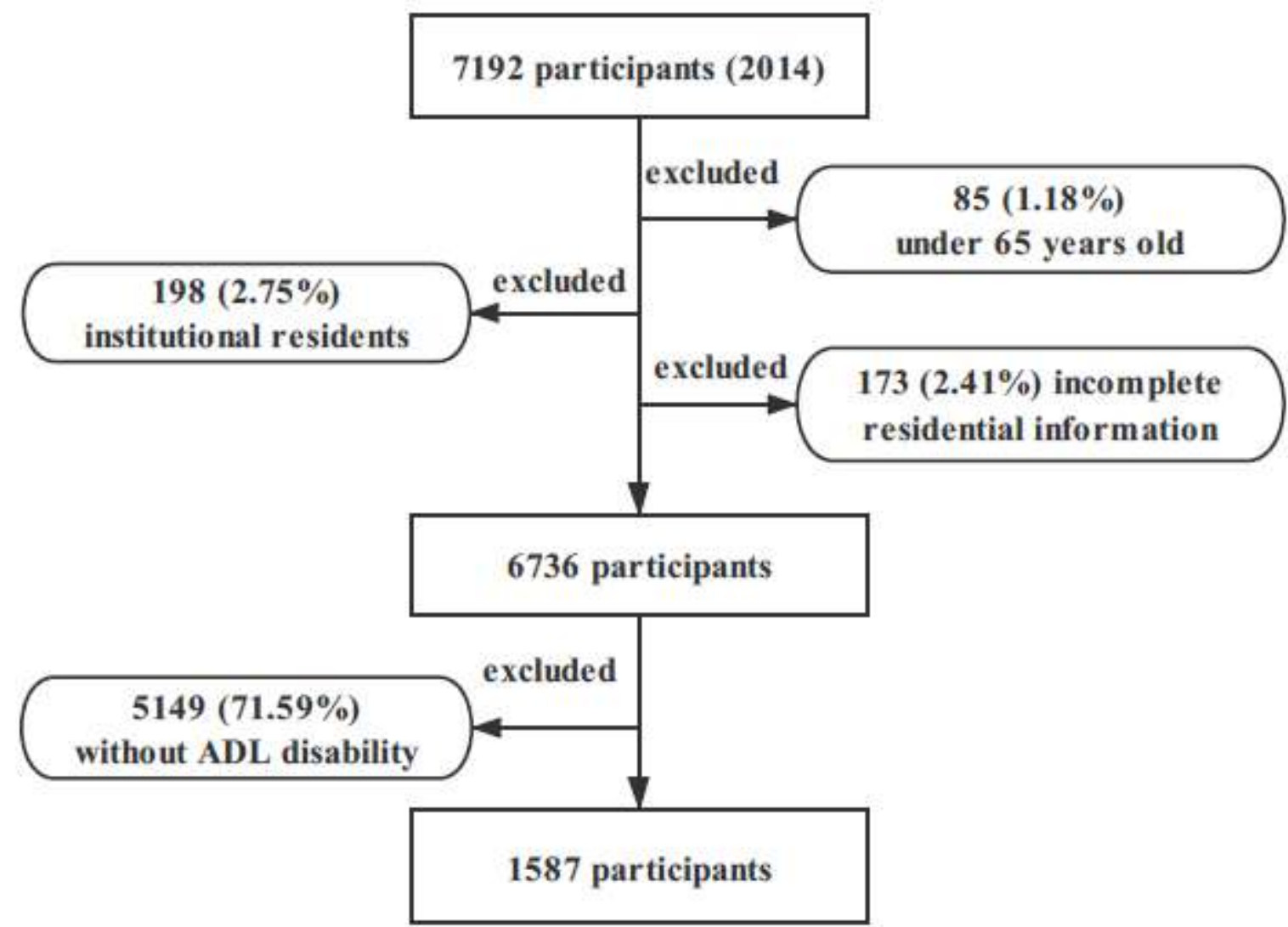

Figure 1. Flow diagram of included and excluded participants 
Figures

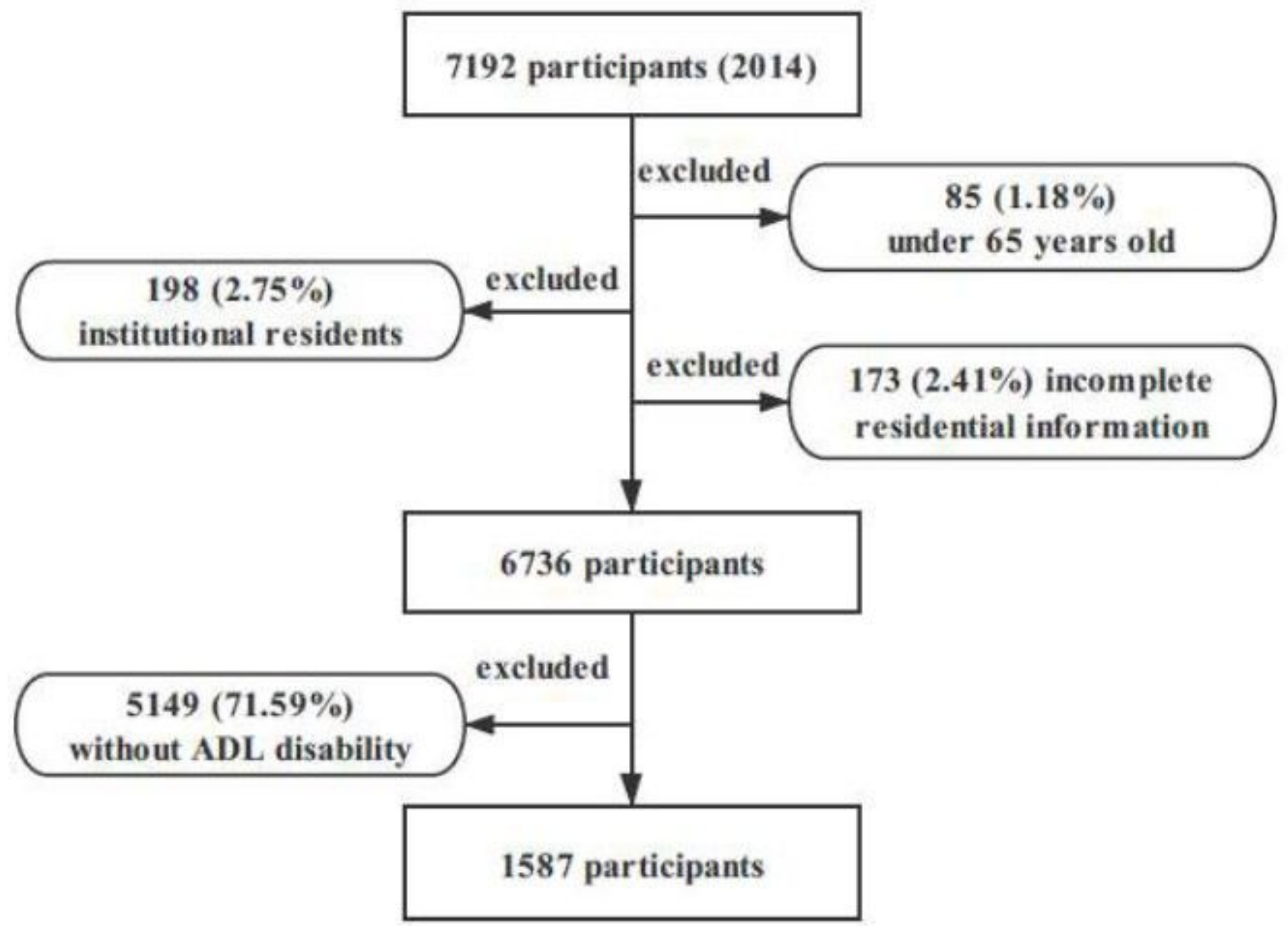

Figure 1

Flow diagram of included and excluded participants 